\title{
Conhecimento da equipe de Enfermagem acerca do processo transfusional na
}

\section{Unidade de Terapia Intensiva}

\author{
Knowledge of the Nursing team about the transfusion process in the Intensive Care Unit \\ Conocimiento del equipo de Enfermería sobre el proceso de transfusión en la Unidad de Cuidados
}

Intensivos

Recebido: 19/01/2022 | Revisado: 27/01/2022 | Aceito: 03/02/2022 | Publicado: 05/02/2022

\author{
Laudivania Claudio de Andrade \\ ORCID: https://orcid.org/0000-0001-5995-1933 \\ Universidade Estadual de Ciências da Saúde de Alagoas, Brasil \\ E-mail: laudivaniaandrade@outlook.com \\ Irena Penha Duprat \\ ORCID: https://orcid.org/0000-0001-8273-4822 \\ Universidade Estadual de Ciências da Saúde de Alagoas, Brasil \\ E-mail: irena.penha@gmail.com \\ Cristiane Maria Alves Martins \\ ORCID: https://orcid.org/0000-0001-6804-7014 \\ Universidade Estadual de Ciências da Saúde de Alagoas, Brasil \\ E-mail: cmamartins@gmail.com \\ Amanda Cavalcante de Macedo \\ ORCID: https://orcid.org/0000-0002-4630-2771 \\ Universidade Estadual de Ciências da Saúde de Alagoas, Brasil \\ E-mail: amandacmacedo@gmail.com \\ Janine Melo de Oliveira \\ ORCID: https://orcid.org/0000-0002-8816-2244 \\ Universidade Estadual de Ciências da Saúde de Alagoas, Brasil \\ E-mail: nine.melo@hotmail.com
}

\begin{abstract}
Resumo
Objetivo: descrever o conhecimento da equipe de enfermagem acerca do processo transfusional na Unidade de Terapia Intensiva. Metodologia: estudo descritivo transversal, com abordagem quantitativa. Participaram enfermeiros, técnicos de enfermagem e auxiliares de enfermagem que trabalhavam na Unidade de Terapia Intensiva de um hospital geral que é referência para o atendimento de urgência e emergência. Resultados: $44(80,0 \%)$ dos profissionais referiram não ter recebido treinamento prévio em hemoterapia, $39(70,9 \%)$ afirmaram que não participam periodicamente de treinamentos que abordam aspectos como ato transfusional e reações adversas e, $12(21,9 \%)$ mencionaram conhecer as portarias que tratam sobre os Regulamentos Técnicos de Procedimentos Hemoterápicos. Anemia $(56,9 \%)$ e hemorragia $(15,4 \%)$ foram as respostas mais citadas como indicação para transfusão do concentrado de hemácias. Coagulopatias $(35,3 \%)$ e plaquetopenia $(57,5 \%)$ foram as indicações mais referidas para a infusão de plasma fresco e concentrado de plaquetas. Calafrio $(69,1 \%)$, prurido $(60,0 \%)$, sudorese $(52,7 \%)$, taquicardia $(50,9 \%)$ e desconforto respiratório $(49,1 \%)$ foram os sinais e sintomas sugestivos de reações transfusionais mais citados. Apenas $2(3,6 \%)$ participantes mencionaram corretamente o tempo de início de possíveis complicações. Conclusão: evidenciou-se a deficiência no conhecimento dos profissionais da equipe de enfermagem sobre hemotransfusão, bem como o impacto negativo gerado pela falta de treinamentos e orientação sobre a temática no conhecimento dos profissionais. Salienta-se a importância da necessidade de intervenções como educação continuada e permanente, bem como o treinamento periódico dos profissionais da equipe de enfermagem sobre atuação nesta prática.
\end{abstract}

Palavras-chave: Assistência de enfermagem; Conhecimento; Enfermagem; Transfusão sanguínea.

\begin{abstract}
Objective: to describe the knowledge of the nursing team about the transfusion process in the Intensive Care Unit. Methodology: a descriptive cross-sectional study, with a quantitative approach. Participated nurses, nursing technicians and nursing assistants who worked in the Intensive Care Unit of a general hospital that is a reference for urgent and emergency care. Results: 44 (80.0\%) of the professionals reported not having received previous training in hemotherapy, $39(70.9 \%)$ stated that they do not participate periodically in training that addresses aspects such as transfusion and adverse reactions, and $12(21.9 \%)$ mentioned knowing the ordinances dealing with the Technical Regulations for Hemotherapeutic Procedures. Anemia (56.9\%) and hemorrhage (15.4\%) were the most cited responses as an indication for transfusion of red blood cell concentrate. Coagulopathies (35.3\%) and thrombocytopenia (57.5\%) were the most
\end{abstract}


common indications for the infusion of fresh and concentrated platelet plasma. Chills (69.1\%), itching (60.0\%), sweating $(52.7 \%)$, tachycardia $(50.9 \%)$ and respiratory distress $(49.1 \%)$ were the most suggestive signs and symptoms of transfusion reactions cited. Only $2(3.6 \%)$ participants correctly mentioned the time of onset of possible complications. Conclusion: there was a deficiency in the knowledge of the nursing team professionals about blood transfusion, as well as the negative impact generated by the lack of training and guidance on the subject in the professionals' knowledge. It emphasizes the importance of the need for interventions such as continuing and permanent education, as well as the periodic training of professionals of the nursing team on performance in this practice.

Keywords: Nursing assistance; Knowledge; Nursing; Blood transfusion.

\section{Resumen}

Objetivo: describir el conocimiento del equipo de enfermería sobre el proceso de transfusión en la Unidad de Cuidados Intensivos. Metodología: estudio descriptivo transversal, con enfoque cuantitativo. Los participantes fueron enfermeros, técnicos de enfermaría y auxiliares de enfermería que laboraron en la Unidad de Cuidados Intensivos de un hospital general que es un referente en atención de urgencia y emergencia. Resultados: 44 (80,0\%) de los profesionales refirieron no haber recibido formación previa en hemoterapia, 39 (70,9\%) manifestaron no participar periódicamente en capacitaciones que aborden aspectos como transfusión y reacciones adversas, y $12(21,9 \%)$ mencionaron conociendo las ordenanzas relativas al Reglamento Técnico de Procedimientos Hemoterapéuticos. La anemia (56,9\%) y la hemorragia $(15,4 \%)$ fueron las respuestas citadas con mayor frecuencia como indicación de transfusión de concentrado de hematíes. Las coagulopatías $(35,3 \%)$ y la trombocitopenia $(57,5 \%)$ fueron las indicaciones más frecuentes para la infusión de plasma plaquetario fresco y concentrado. Escalofríos $(69,1 \%)$, prurito $(60,0 \%)$, sudoración (52,7\%), taquicardia $(50,9 \%)$ y dificultad respiratoria $(49,1 \%)$ fueron los signos y síntomas más sugestivos de las reacciones transfusionales citadas. Solo 2 (3,6\%) participantes mencionaron correctamente el momento de aparición de las posibles complicaciones. Conclusión: se evidenció la deficiencia en el conocimiento de los profesionales del equipo de enfermería sobre la transfusión sanguínea, así como el impacto negativo que genera la falta de formación y orientación sobre el tema en el conocimiento de los profesionales. Destaca la importancia de la necesidad de intervenciones como la educación continua y permanente, así como la formación periódica de los profesionales del equipo de enfermería sobre el desempeño en esta práctica.

Palabras clave: Asistencia de enfermería; Conocimiento; Enfermería; Transfusión sanguínea.

\section{Introdução}

$\mathrm{Na}$ antiguidade a hemoterapia estava ligada diretamente as religiões. A representação do sangue provocou nos pesquisadores curiosidade, que em conjunto com as práticas empíricas de cura e cultos religiosos colaboraram com o avanço científico da transfusão de sangue e dessa maneira para a hemoterapia conhecida atualmente. Este procedimento objetiva ajudar inúmeros pacientes, do mesmo modo que aprimora os reservatórios dos bancos de sangue. Desse modo, deve ser realizada em circunstancias seguras, mediante profissionais capacitados e com recursos essenciais para atuar nas possíveis intercorrências que venham a proceder (Benites, 2013; Silva et al., 2009).

No Brasil, a normatização dos procedimentos de hemoterapia é determinada pela Portaria $n^{\circ} 158$, publicada pelo Ministério da Saúde (MS), em 04 de fevereiro de 2016, que redefine o regulamento técnico de procedimentos hemoterápicos de acordo com os princípios e diretrizes da Política Nacional de Sangue, Componentes e Derivados. Além das regras que devem ser conhecidas e seguidas pelos profissionais que atuam em hemotransfusão, esta portaria estabelece que os locais de procedimento devem estar providos de equipamentos essenciais para atender aos pacientes em casos de reações adversas, por esse motivo, na prática assistencial, a hemotransfusão ocorre com maior frequência nas Unidades de Terapia Intensiva (UTIs), devido à gravidade dos pacientes (Brasil, 2016; Silva et al., 2009).

A Enfermagem tem papel importante na garantia da segurança transfusional, pois a equipe é responsável por conhecer as indicações de transfusões, realizar a checagem de dados para prevenir erros, orientar os pacientes sobre a hemotransfusão, detectar e atuar no atendimento às reações transfusionais e documentar o procedimento. Tendo em vista toda a complexidade deste procedimento e a necessidade de conhecimentos específicos em todo o seu desenvolvimento, o Conselho Federal de Enfermagem através da Resolução n 629/2020, aprovou e atualizou a Norma Técnica que dispõe sobre a Atuação de Enfermeiro e de Técnico de Enfermagem em Hemoterapia. Este documento tem como finalidade estabelecer diretrizes para a atuação destes profissionais em Hemoterapia a fim de garantir uma assistência eficiente, segura e de qualidade (Conselho 
Federal de Enfermagem [COFEN], 2020; Mattia \& Andrade, 2016).

Embora a Enfermagem desempenhe papel essencial na hemoterapia, ainda é escasso o número de publicações realizadas pela categoria sobre esta temática. Estudos evidenciam que muitos profissionais sequer receberam capacitação sobre hemotransfusão não tendo, portanto, o conhecimento e segurança necessários para atuar na realização do procedimento. Alguns profissionais sabem realizar toda a técnica do procedimento, mas não possuem base cientifica e habilidade suficientes para reconhecer e atuar nas reações transfusionais (Brazeiro et al., 2021; Conceição, 2015).

Neste contexto, julgou-se relevante desenvolver um estudo com enfoque na realização do procedimento de hemotransfusão pela equipe de enfermagem na UTI, visto ser este um setor em que a hemotransfusão acontece com grande frequência, sendo importante o respeito aos aspectos de segurança do paciente. Desse modo, o estudo se destina a descrever o conhecimento da equipe de enfermagem acerca do processo transfusional na Unidade de Terapia Intensiva (UTI), tornando-se relevante por descrever se a equipe de enfermagem, em suas atribuições, está preparada para atuar no processo transfusional desempenhando a assistência de enfermagem de acordo com as resoluções preconizadas e, apresenta a seguinte questão norteadora: como se apresenta o conhecimento da equipe de enfermagem acerca do processo transfusional na UTI?

\section{Metodologia}

Trata-se de uma pesquisa de campo, de natureza quantitativa, transversal e descritiva (f, 2018). O estudo foi desenvolvido em uma Unidade de Terapia Intensiva adulto de um hospital geral que é referência para o atendimento de urgência e emergência, localizado na cidade de Maceió - AL.

A equipe de enfermagem da UTI é composta por 67 profissionais, destes $55(82,1 \%)$ foram selecionados para participar do estudo por amostragem não probabilística, elegíveis dentro dos critérios de inclusão e exclusão, sendo 10 enfermeiros, 39 técnicos de enfermagem e 06 auxiliares de enfermagem que trabalhavam nos três turnos (manhã, tarde e noite) na referida UTI.

Foram incluídos enfermeiros, técnicos de enfermagem e auxiliares de enfermagem que trabalhem na UTI, que já tivessem atendido pacientes recebendo transfusão, e que tivesse no mínimo três meses de experiência no setor. Foram excluídos os enfermeiros, técnicos de enfermagem e auxiliares de enfermagem que estavam afastados do serviço durante o período de coleta de dados, por motivo de férias, licença médica ou maternidade.

A coleta de dados foi realizada pela pesquisadora entre os meses de julho a outubro de 2018, durante o turno de trabalho dos profissionais de enfermagem, conforme sua disponibilidade, e em um lugar reservado.

Os dados foram obtidos por meio de um instrumento próprio padronizado, contendo perguntas referentes aos dados sóciodemográficos e outras direcionadas sobre a temática pesquisada que permitiram responder os objetivos propostos. Os participantes foram requisitados uma única vez e antes de responderem aos questionários em si, foram assinados, por extenso, os Termos de consentimento livre esclarecido - TCLE, em duas vias idênticas, sendo uma delas entregue ao participante e a outra a pesquisadora. $\mathrm{O}$ sigilo e anonimato dos sujeitos foram garantidos através da identificação numérica e relacionados em uma lista.

Os dados foram ordenados em forma de planilha no "software" Excel for Windows, versão 2013, utilizando a variável primária "conhecimento a acerca do processo transfusional" e as variáveis secundarias: sexo; faixa etária; tempo de serviço na UTI; função exercida na instituição; e nível de formação profissional. Os resultados foram tabulados e apresentados na forma descritiva através de tabelas e figuras.

A pesquisa obedeceu aos critérios éticos estabelecidos pelo conselho de saúde brasileiro e foi aprovada pelo Comitê de Ética em Pesquisa (CEP) da Universidade Estadual de Ciências da Saúde de Alagoas sob registro de nº 90108418.7.0000.5011. 


\section{Resultados e Discussão}

A amostra foi constituída por 55 profissionais da equipe de enfermagem que representam 82,1\% do total de profissionais de enfermagem (67) da UTI, sendo a maior categoria encontrada a de técnicos de enfermagem (70,9\%), também em consonância com a literatura; essa classe geralmente é maior, por configurar-se como a contingente da força de trabalho da equipe de enfermagem (Souza et al., 2014).

A Tabela 1 apresenta a distribuição dos profissionais de enfermagem de acordo com o sexo, faixa etária e tempo de serviço na UTI. Houve um predomínio de profissionais do sexo feminino (90,0\%), faixa etária entre 30 a 44 anos (54,6\%) e entre 10 e 19 anos (40,0\%) de serviço na UTI da instituição. Dados semelhantes também foram encontrados por E. B. Ferreira et al. (2021) e Lopes e Leal (2005) havendo a predominância feminina em todas as categorias de trabalhadores de enfermagem, afirmando que a enfermagem é uma profissão exercida, em sua maioria, por mulheres. Quanto ao tempo de serviço na unidade pesquisada, dados similares foram encontrados por Carneiro et al. (2017) havendo a predominância de mais de 10 anos de trabalho na unidade onde ocorreu a pesquisa.

Tabela 1. Distribuição dos profissionais de enfermagem de acordo com o sexo, faixa etária e tempo de serviço na UTI, segundo a função exercida na instituição. Maceió, AL, 2019.

\begin{tabular}{|c|c|c|c|c|c|c|c|c|}
\hline \multicolumn{9}{|c|}{ Função exercida na instituição } \\
\hline & \multicolumn{2}{|c|}{$\begin{array}{c}\text { Auxiliar de } \\
\text { Enfermagem }\end{array}$} & \multicolumn{2}{|c|}{$\begin{array}{c}\text { Técnico de } \\
\text { Enfermagem }\end{array}$} & \multicolumn{2}{|c|}{ Enfermeiro } & \multicolumn{2}{|c|}{ Total } \\
\hline & $\mathrm{n}$ & $\%$ & $\mathrm{n}$ & $\%$ & $\mathrm{n}$ & $\%$ & $\mathrm{n}$ & $\%$ \\
\hline \multicolumn{9}{|l|}{ Sexo } \\
\hline Feminino & 06 & 10,9 & 35 & 63,6 & 09 & 16,4 & 50 & 90,9 \\
\hline Masculino & 00 & 00 & 04 & 7,3 & 01 & 1,8 & 05 & 9,1 \\
\hline Total & 06 & 10,9 & 39 & $\mathbf{7 0 , 9}$ & 10 & 18,2 & 55 & 100 \\
\hline \multicolumn{9}{|l|}{ Faixa etária (anos) } \\
\hline$\leq 29$ & 00 & 00 & 03 & 5,4 & 01 & 1,8 & 04 & 7,3 \\
\hline 30 a 44 & 04 & 7,3 & 20 & 36,4 & 06 & 10,9 & 30 & 54,6 \\
\hline$\geq 45$ & 02 & 3,6 & 16 & 29,1 & 03 & 5,4 & 21 & 38,1 \\
\hline Total & 06 & 10,9 & 39 & 70,9 & 10 & 18,2 & 55 & 100 \\
\hline \multicolumn{9}{|c|}{ Tempo de serviço na UTI (anos) } \\
\hline$\leq 01$ & 00 & 00 & 05 & 9,1 & 00 & 00 & 05 & 9,1 \\
\hline$>01$ a 09 & 00 & 00 & 13 & 23,7 & 06 & 10,9 & 19 & 34,6 \\
\hline 10 a 19 & 04 & 7,3 & 16 & 29,1 & 02 & 3,6 & 22 & 40,0 \\
\hline$\geq 20$ & 02 & 3,6 & 03 & 5,4 & 01 & 1,8 & 06 & 10,9 \\
\hline Não respondeu & 00 & 00 & 02 & 3,6 & 01 & 1,8 & 03 & 5,4 \\
\hline Total & 06 & 10,9 & 39 & 70,9 & 10 & 18,2 & 55 & 100 \\
\hline
\end{tabular}

Fonte: Dados da pesquisa.

A Tabela 2 representa a distribuição dos profissionais de acordo com a sua formação profissional na área de enfermagem. Observa-se que dos 55 entrevistados, 21 (38,1\%) possuíam formação em mais de uma categoria e que 16 (29,0\%) iniciaram sua formação profissional como auxiliar de enfermagem. Dos $21(38,1 \%)$ que tinham curso de graduação em enfermagem, apenas $10(18,2 \%)$ exerciam a função de enfermeiro no serviço de UTI.

De acordo com a Lei n. ${ }^{\circ}$ 7.498/86, que dispõe sobre a regulamentação do exercício da Enfermagem e dá outras providências, a organização e divisão do trabalho em enfermagem no Brasil estão subdivididas em categorias profissionais, sendo elas: enfermeiro, técnico de enfermagem e auxiliar de enfermagem (a última não é mais regulamentada em lei, por isto está em extinção). Nestas subdivisões, o técnico e o auxiliar de enfermagem exercem atividades de nível médio e de baixa complexidade e ao Enfermeiro é atribuída todas as atividades de enfermagem e atividades de maior complexidade técnica 
(privativas do enfermeiro) (Conselho Federal de Enfermagem [COFEN], 1986).

Ao analisar o crescimento da força de trabalho em enfermagem se constata que, majoritariamente, o número de técnicos de enfermagem cresceu de forma mais acentuada, devido ao incremento das políticas educacionais de profissionalização na área da saúde. Entretanto, os técnicos e auxiliares de enfermagem cursam a graduação por esta possibilitar uma diversidade de lócus de atuação em múltiplos cenários, espaços e contextos do atendimento à saúde. Também por ser um curso menos seletivo que outros considerados de maior prestígio social, por permitir a ascensão profissional, por programar o conhecimento científico e por vislumbrar mudança de status dentro da equipe (Costa et al., 2008; Fontana \& Brigo, 2011).

Tabela 2. Profissionais de acordo com o nível de formação profissional na área de enfermagem. Maceió, AL, 2019.

\begin{tabular}{lll}
\hline Nível de formação profissional & $\mathbf{n}$ & $\%$ \\
\hline $\mathrm{AE}$ & 01 & 1,8 \\
$\mathrm{AE}+\mathrm{E}$ & 02 & 3,6 \\
$\mathrm{AE}+\mathrm{TE}$ & 10 & 18,2 \\
$\mathrm{AE}+\mathrm{TE}+\mathrm{E}$ & 03 & 5,4 \\
$\mathrm{E}$ & 10 & 18,2 \\
TE & 23 & 41,9 \\
TE + E & 06 & 10,9 \\
\hline Total & $\mathbf{5 5}$ & $\mathbf{1 0 0}$ \\
\hline
\end{tabular}

AE: Auxiliar de Enfermagem TE: Técnico de Enfermagem E: Enfermeiro. Fonte: Dados da pesquisa.

Partindo-se da variável conhecimento acerca do processo transfusional e correlacionando as variáveis secundárias, observa-se que 44 (80,0\%) dos sujeitos da pesquisa referiram não ter recebido treinamento prévio em hemoterapia e 39 (70,9\%) afirmaram que não participam periodicamente de treinamentos que abordam aspectos como ato transfusional e reações adversas. Vale destacar que o Enfermeiro assume papel importante no que diz respeito ao ato transfusional. No entanto, apenas $1(1,8 \%)$ afirmou ter recebido treinamento prévio, porém negaram participar de treinamentos periódicos sobre a temática (Tabela 3). $\mathrm{O}$ estudo de Tavares et al. (2015), discordando com os achados dessa pesquisa, revelou que a maioria dos profissionais recebeu treinamento ou orientação para realizar o processo transfusional.

Outra pesquisa identificou que os profissionais de enfermagem se sentiam despreparados por não participarem de treinamentos sobre o assunto, com destaque aos enfermeiros entrevistados que afirmaram nunca ter participado de treinamentos sobre hemoterapia na instituição onde ocorreu a pesquisa, corroborando os achados do presente estudo (Vieira, 2012). 
Tabela 3. Distribuição dos profissionais de enfermagem de acordo com tipo de informação recebida sobre procedimentos hemoterápicos, segundo a função exercida na instituição. Maceió, AL, 2019.

\begin{tabular}{|c|c|c|c|c|c|c|c|c|}
\hline & \multicolumn{8}{|c|}{ Função exercida na instituição } \\
\hline & \multicolumn{2}{|c|}{$\begin{array}{c}\text { Auxiliar de } \\
\text { Enfermagem }\end{array}$} & \multicolumn{2}{|c|}{$\begin{array}{c}\text { Técnico de } \\
\text { Enfermagem }\end{array}$} & \multicolumn{2}{|c|}{ Enfermeiro } & \multicolumn{2}{|c|}{ Total } \\
\hline & $\mathrm{n}$ & $\%$ & $\mathrm{n}$ & $\%$ & $\mathrm{n}$ & $\%$ & $\mathrm{n}$ & $\%$ \\
\hline \multicolumn{9}{|l|}{ Recebeu treinamento prévio } \\
\hline Sim & 01 & 1,8 & 08 & 14,6 & 01 & 1,8 & 10 & 18,2 \\
\hline Não & 05 & 9,1 & 30 & 54,5 & 09 & 16,4 & 44 & 80,0 \\
\hline Não desejo responder & 00 & 00 & 01 & 1,8 & 00 & 00 & 01 & 1,8 \\
\hline Total & 06 & 10,9 & 39 & 70,9 & 10 & 18,2 & 55 & 100 \\
\hline \multicolumn{9}{|l|}{$\begin{array}{l}\text { Participa periodicamente de } \\
\text { treinamentos }\end{array}$} \\
\hline Sim & 02 & 3,6 & 12 & 21,9 & 00 & 00 & 14 & 25,5 \\
\hline Não & 04 & 7,3 & 25 & 45,4 & 10 & 18,2 & 39 & 70,9 \\
\hline Não desejo responder & 00 & 00 & 02 & 3,6 & 00 & 00 & 02 & 3,6 \\
\hline Total & 06 & 10,9 & 39 & 70,9 & 10 & 18,2 & 55 & 100 \\
\hline
\end{tabular}

Fonte: Dados da pesquisa.

Quanto ao conhecimento dos profissionais de enfermagem sobre as portarias que tratam sobre os Regulamentos Técnicos de Procedimentos Hemoterápicos, apenas 12 (21,9\%) afirmaram conhecê-las, evidenciando um déficit no conhecimento sobre hemoterapia. Pesquisa realizada por Silva et al. (2009), revelou que apenas 4 (14,8\%) dos profissionais relataram conhecer a resolução vigente no período da pesquisa, corroborando os achados do presente estudo.

Estudos enfatizam que profissionais sem capacidade técnica suficiente e sem conhecimentos em hemoterapia podem diminuir a segurança transfusional e causar danos graves ao paciente. Deste modo, a atuação competente torna-se requisito essencial para segurança do paciente, prevenindo as possíveis complicações e reações transfusionais (O. Ferreira et al., 2007).

Segundo Conselho Federal de Enfermagem (COFEN, 2020) a equipe de enfermagem em hemoterapia é formada por enfermeiros e técnicos de enfermagem, executando estes profissionais suas atribuições em conformidade com o disposto na legislação. Por ser considerada uma terapia de alta complexidade, é vedada aos auxiliares de enfermagem a execução de ações relacionadas à hemoterapia podendo, no entanto, executar cuidados de higiene e conforto ao paciente. Contudo, o presente estudo evidenciou a participação de auxiliares de enfermagem na realização dos procedimentos hemoterápicos, evidenciando o descumprimento das leis vigentes no país.

Partindo desta realidade, segundo Brasil (2016) os profissionais que atuam em hemoterapia devem conhecer as regras deste procedimento e segui-las para que este ocorra de forma segura evitando riscos e garantindo a segurança do paciente.

Em razão disso, é de suma importância que o profissional de enfermagem se mantenha sempre atualizado cientificamente sobre questões específicas como, por exemplo, a hemoterapia. Sendo importante que este conheça as portarias que orienta e normatiza as atividades que o mesmo realiza, tendo assim respaldo legal e segurança em suas atividades.

Analisando as questões que abordam o conhecimento da equipe de enfermagem sobre as indicações para transfusão dos hemocomponentes, constatou-se que $40(80,0 \%)$ dos profissionais afirmaram conhecer pelo menos uma indicação para transfusão do concentrado de hemácias $(\mathrm{CH})$. E dentre elas, anemia $(56,9 \%)$ e hemorragia $(15,4 \%)$ foram as repostas mais citadas. Apesar de afirmarem conhecer, 20 (11,3\%) não citaram exemplos. Coagulopatias (35,3\%) e plaquetopenia (57,5\%) foram às indicações mais referidas para a infusão de plasma fresco (PF) e concentrado de plaquetas (CP), respectivamente, como está demonstrado na Tabela 4.

Segundo Silva et al. (2009), as transfusões sanguíneas ocorrem com grande frequência nas UTIs, por esta razão os profissionais de enfermagem atuantes nestes setores devem conhecer as indicações para o uso dos componentes sanguíneos. No 
entanto, evidenciou-se um déficit neste conhecimento, pois embora o $\mathrm{CH}$ tenha sido o percentual maior, houve um baixo percentual nas indicações para o uso de PF e CP, sendo importante que todos soubessem.

Tabela 4. Conhecimento referido pela equipe de enfermagem sobre indicações da hemotransfusão. Maceió, AL, 2019.

\begin{tabular}{|c|c|c|c|c|c|c|c|c|}
\hline \multirow{2}{*}{$\begin{array}{l}\text { Conhecimento referido sobre indicações de } \\
\text { transfusão de componentes sanguíneos* }\end{array}$} & \multicolumn{2}{|c|}{ Auxiliar } & \multicolumn{2}{|c|}{ Técnico } & \multicolumn{2}{|c|}{ Enfermeiro } & \multicolumn{2}{|c|}{ Total } \\
\hline & $\mathrm{n}$ & $\%$ & $\mathrm{n}$ & $\%$ & $\mathrm{n}$ & $\%$ & $\mathrm{n}$ & $\%$ \\
\hline \multicolumn{9}{|l|}{ Concentrado de Hemácias*** } \\
\hline Anemia & 07 & 10,8 & 22 & 33,8 & 08 & 12,3 & 37 & 56,9 \\
\hline Choque & 02 & 3,1 & 05 & 7,7 & 01 & 1,5 & 08 & 12,3 \\
\hline Hemorragia & 00 & 00 & 10 & 15,4 & 00 & 00 & 10 & 15,4 \\
\hline Plaquetopenia & 01 & 1,5 & 04 & 6,2 & 01 & 1,5 & 06 & 9,2 \\
\hline Outros & 01 & 1,5 & 02 & 3,1 & 01 & 1,5 & 04 & 6,2 \\
\hline Total & 11 & 16,9 & 43 & 66,2 & 11 & 16,9 & 65 & 100 \\
\hline \multicolumn{9}{|l|}{ Plasma Fresco ${ }^{* * *}$} \\
\hline Coagulopatias & 01 & 2,9 & 07 & 20,6 & 04 & 11,8 & 12 & 35,3 \\
\hline Hemorragia & 01 & 2,9 & 06 & 17,6 & 02 & 5,9 & 09 & 26,4 \\
\hline Hipoalbuminemia & 00 & 00 & 00 & 00 & 02 & 5,9 & 02 & 5,9 \\
\hline Leucemia & 00 & 00 & 02 & 5,9 & 00 & 00 & 02 & 5,9 \\
\hline Outros & 01 & 2,9 & 04 & 11,8 & 02 & 5,9 & 07 & 20,6 \\
\hline Não se aplica & 00 & 00 & 02 & 5,9 & 00 & 00 & 02 & 5,9 \\
\hline Total & $\mathbf{0 3}$ & 8,7 & 21 & 61,8 & 10 & 29,5 & 34 & 100 \\
\hline \multicolumn{9}{|l|}{ Concentrado de Plaquetas $* * * *$} \\
\hline Hemorragia & 01 & 2,1 & 02 & 4,3 & 00 & 00 & 03 & 6,4 \\
\hline Plaquetopenia & 02 & 4,3 & 19 & 40,4 & 06 & 12,8 & 27 & 57,5 \\
\hline Leucemia & 01 & 2,1 & 01 & 2,1 & 02 & 4,3 & 04 & 8,5 \\
\hline Distúrbio de coagulação & 01 & 2,1 & 06 & 12,8 & 00 & 00 & 07 & 14,9 \\
\hline Outros & 00 & 00 & 04 & 8,5 & 01 & 2,1 & 05 & 10,6 \\
\hline Não se aplica & 00 & 00 & 01 & 2,1 & 00 & 00 & 01 & 2,1 \\
\hline Total & 05 & 10,6 & 33 & 70,2 & 09 & 19,2 & 47 & 100 \\
\hline
\end{tabular}

*O participante poderia descrever mais de uma resposta. $* *$ n: $65 * * * n: 34 * * * n$ : 47 . Fonte: Dados da pesquisa.

Sobre as etapas realizadas pelos profissionais de enfermagem no ato transfusional, 41 (74,5\%) afirmaram que verificam os sinais vitais antes do início da transfusão, 37 (67,2\%) registram os sinais vitais verificados antes do início da transfusão, 51 $(92,7 \%)$ conferem a identificação da bolsa que irá ser transfundida com os dados do receptor, 24 (43,6\%) permanecem ao lado do paciente nos primeiros 10 minutos iniciais da transfusão, $38(69,1 \%)$ monitoram o paciente periodicamente durante o transcurso do ato transfusional e $32(58,1 \%)$ verificam os sinais vitais após o término da transfusão.

As afirmações supracitadas seguem as recomendações da Portaria n 158/2016 do Ministério da Saúde que redefine o regulamento técnico de procedimentos hemoterápicos a qual descreve na Seção VII as etapas a serem realizadas no ato transfusional (Brasil, 2016). Apesar de todas as etapas serem recomendadas pela legislação e algumas serem atividades básicas do cotidiano da equipe de enfermagem, percebe-se que nem todos os participantes as executam, demonstrando assim não possuírem o hábito de realizar tais atividades durante as transfusões sanguíneas, podendo colocar em risco a integridade do ato transfusional para o paciente.

Acerca do conhecimento dos pesquisados sobre o tempo de permanência dos componentes eritrocitários em temperatura ambiente antes do início da sua infusão observa-se, que menos da metade, $25(45,4 \%)$ dos pesquisados afirmaram ser de no máximo 30 minutos. Sobre o tempo máximo de infusão de um hemocomponente, $44(80,0 \%)$ profissionais assinalaram de modo correto que este tempo não deve exceder 04 horas.

Já em relação ao procedimento a ser adotado, caso o tempo máximo de infusão seja excedido, 40 (72,7\%) entrevistados indicaram a suspensão do hemocomponente e o descarte da bolsa em local apropriado. 
Segundo a Portaria n ${ }^{\circ} 158$ do MS, antes do início da transfusão, os componentes eritrocitários não permanecerão em temperatura ambiente por mais de 30 minutos e os componentes sanguíneos serão infundidos em, no máximo, 4 horas, caso este período estabelecido for atingindo, a transfusão será interrompida e as bolsas descartadas (Brasil, 2016).

Para o tempo máximo de infusão e as medidas caso o tempo seja excedido, houve um bom percentual de respostas condizentes com as recomendações da portaria o que não se observa em relação ao tempo de permanência em temperatura ambiente antes da infusão.

Outro ponto de destaque na pesquisa e que merece maior atenção relaciona-se a complicação transfusional imediata, pois apenas 2 (3,6\%) dos participantes mencionaram o tempo de início de possíveis complicações corretamente. De acordo com a Portaria $n^{\circ} 158$ (Brasil, 2016) consideram-se reações transfusionais imediatas aquelas que ocorrem até 24 horas depois de iniciada a transfusão.

Segundo Brasil (2015), Fidlarczyk e Ferreira (2008) e Silva et al. (2009), todos os profissionais de enfermagem envolvidos na assistência ao paciente que recebe transfusão sanguínea devem estar capacitados a saber identificar quaisquer sinais e sintomas que indiquem a ocorrência de uma reação transfusional e também os cuidados e procedimentos adequados para uma assistência de qualidade especialmente os profissionais que atuam nas UTIs, pois esses setores hospitalares apresentam as maiores frequências de procedimentos hemoterápicos.

Dentre as medidas adequadas a serem adotadas em casos de reações transfusionais imediatas, 18 (32,8\%) afirmaram interromper a transfusão, sendo que em casos de reações alérgicas leves (urticárias) a transfusão não precisa ser suspensa, 12 (21,9\%) mantém o acesso venoso, 36 (64,4\%) examinam os rótulos da bolsa e todos os registros atinentes para verificar se houve erro na identificação do paciente ou das bolsas transfundidas, 33 (60,0\%) verificam os sinais vitais e 41 (74,5\%) comunicam ao médico assistente e/ou médico do serviço de hemoterapia.

Apesar de todas as medidas estarem corretas, nota-se que as mesmas não são frequentemente realizadas nas condutas adotadas pelos profissionais frente às adversidades transfusionais o que é preocupante, pois é importante que todos profissionais envolvidos na administração de hemocomponentes estejam capacitados e preparados a identificar e utilizar estratégias adequadas para resolução e prevenção dos episódios de reação transfusional (Brasil, 2015).

A Figura 1 apresenta os sinais e sintomas sugestivos de reações transfusionais de acordo com as respostas dos entrevistados, as mais citadas foram: calafrio $(69,1 \%)$; prurido $(60,0 \%)$; sudorese $(52,7 \%)$; taquicardia $(50,9 \%)$; e desconforto respiratório $(49,1 \%)$. Esses resultados confirmam os achados de um estudo realizado por Carneiro et al. (2017), que teve dentre os sinais e sintomas sugestivos de reações transfusionais, as respostas mais citadas: febre $(62,0 \%)$; prurido (44,8\%); tremor (37,9\%); exantema (34,4\%) e calafrio (34,4\%). Sendo assim podemos afirmar que identificar os sinais e sintomas nem sempre é fácil, pois as manifestações das reações transfusionais podem se dar de diversas maneiras, como calafrios, febre, urticárias, dispnéias, hipertensão, cefaléia, edemas, taquicardia, dor no peito e na região lombar, eritema, tosse, pápulas, exantema, vômitos, prurido, entre outras, podendo esses não serem percebidos pelos profissionais devido a gravidade do estado de saúde que se encontram os pacientes admitidos nas UTIs (Conceição, 2015).

Apesar da diversidade de sintomas advindos de uma reação transfusional, percebe-se o pouco conhecimento da equipe de enfermagem, principalmente pela pequena quantidade de sintomas citados por cada participante. No entanto, um sintoma comum presente nas reações transfusionais imediatas, que é o calafrio foi citado com bastante frequência. O prurido e sudorese, que também são sintomas comuns foram citados com frequência.

Vale ressaltar que a falta de treinamentos e/ou educação continuada, oferecidos pela instituição sobre essa temática, pode ter influência sobre esse resultado. Dessa maneira, é necessário que a equipe de enfermagem esteja treinada e preparada para detectar precocemente as reações transfusionais para que estes atuem e utilizem as medidas apropriadas o mais precocemente possível. 
Figura 1. Sinais e sintomas das reações transfusionais imediatas citados pelos participantes. Maceió, AL, 2019.

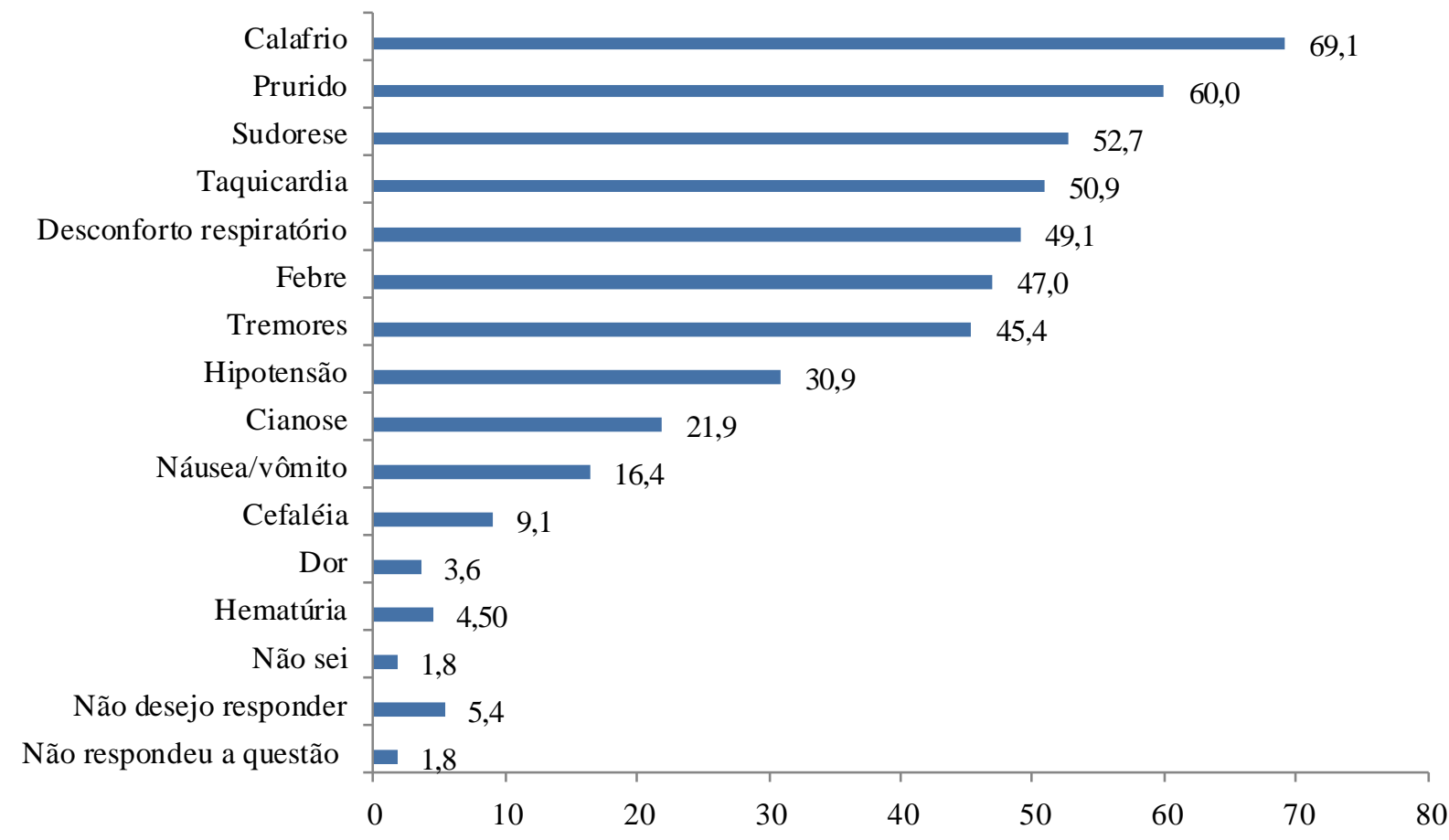

*O participante poderia assinalar mais de uma alternativa. Fonte: Dados da pesquisa.

Uma limitação para a pesquisa foi à inobservância da pratica da administração dos hemocomponentes, tendo em vista o desenho desta investigação, porém não houve prejuízo para o alcance dos objetivos propostos. Recomenda-se a realização de novas pesquisas que objetivem a observação sistemática prática relacionada a hemoterapia considerando a segurança transfusional.

\section{Conclusão}

O desenvolvimento do presente estudo possibilitou descrever o conhecimento da equipe de enfermagem acerca do processo transfusional na UTI.

Evidenciou-se a deficiência no conhecimento dos profissionais da equipe de enfermagem, sobre hemotransfusão, bem como o impacto negativo gerado pela falta de treinamentos e orientação sobre a temática no conhecimento dos profissionais e a participação inadequada dos auxiliares de enfermagem no procedimento. Além disso, forneceu um diagnóstico para a identificação das principais dificuldades dos profissionais com relação às etapas do processo transfusional.

Identificou-se a necessidade de intervenções como educação continuada e permanente, bem como o treinamento periódico dos profissionais da equipe de enfermagem sobre a atuação nesta prática. É importante ressaltar a relevância da realização de capacitações que desenvolvam conhecimentos, habilidades e competências e a aplicação de instrumentos de avaliação do conhecimento, periodicamente. A partir dessas ações, será possível desenvolver uma melhoria na qualidade da execução dos procedimentos e consequente monitoramento da prática.

Esta pesquisa apresenta limitações quanto à população pesquisada, por se tratar de uma amostra limitada e de um único setor de uma mesma instituição, o que influencia nos resultados. Em razão disso, ressalta-se ainda a necessidade de mais pesquisas que se aprofundem no assunto e que sejam realizadas nos demais setores, para que os mesmos possam verificar a pratica de enfermagem frente à administração de hemocomponentes, buscando fomentar reflexões sobre o tema. 
Neste sentido, o conhecimento sobre processo transfusional vai auxiliar as equipes de enfermagem que atuam com hemotransfusões a planejar medidas e atividades que objetivam melhorar, manter e promover a qualidade no atendimento prestado permitindo que estes profissionais realizem seu trabalho de forma mais segura e eficiente, fortalecendo a confiança da equipe e, consequentemente a melhoria no atendimento ao paciente submetido à transfusão.

\section{Agradecimentos}

À Universidade Estadual de Ciências da Saúde de Alagoas (UNCISAL) e ao Fundo de Amparo à Pesquisa do Estado de Alagoas (FAPEAL) pela concessão da bolsa de iniciação científica.

\section{Referências}

Benites, R. M. (2013). A formação em hemoterapia no Rio Grande do Sul: um olhar necessário. Monografia (Especialização) - Curso de Formação Integrada Multidisciplinar em Educação e Ensino em Saúde, Núcleo de Educação, Avaliação e Produção Pedagógica em Saúde, Universidade Federal do Rio Grande do Sul, Porto Alegre. https://www.lume.ufrgs.br/bitstream/handle/10183/115467/000953945.pdf?sequence=1\&isAllowed=y.

Brasil. (2016). Ministério da Saúde. Portaria No 158, de 04 de fevereiro de 2016. Ementa: Redefine o regulamento técnico de procedimentos hemoterápicos. Diário Oficial da União, Poder $\quad$ Executivo, $\quad$ Brasília, $\quad$ DF, $05 \quad$ fev. $2016 . \quad$ (1). $37-100$. http://bvsms.saude.gov.br/bvs/saudelegis/gm/2016/prt0158_04_02_2016.html.

Brasil. (2015). Ministério da Saúde, Secretaria de Atenção à Saúde, Departamento de Atenção Especializada e Temática. Guia para uso de hemocomponentes. 2.ed., 1. reimpr. Brasília: Ministério da Saúde. http://bvsms.saude.gov.br/bvs/publicacoes/guia_uso_hemocomponentes_2ed.pdf.

Brazeiro, L. A., Pereira, P. C., Cardoso, L. S., Scherer, A. C., de Vargas, E., \& Cardoso, L. S. (2021). O Entendimento dos Profissionais de Enfermagem sobre

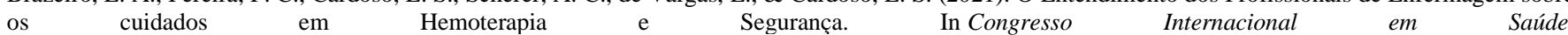
https://publicacoeseventos.unijui.edu.br/index.php/conintsau/article/download/19349/18082.

Carneiro, V. S. M., Barp, M., \& Coelho, M. A. (2017). Hemoterapia e reações transfusionais imediatas: atuação e conhecimento de uma equipe de enfermagem. REME - Rev Min Enferm, 21:e-1031. https://cdn.publisher.gn1.link/reme.org.br/pdf/e1031.pdf.

Conselho Federal de Enfermagem. (2020). Resolução nº 629/2020: Aprova e Atualiza a Norma Técnica que dispõe sobre a Atuação de Enfermeiro e de Técnico de Enfermagem em Hemoterapia. Brasília. http://www.cofen.gov.br/resolucao-cofen-no-629-2020_77883.html.

Conselho Federal de Enfermagem. (1986). Lei n.7498/86, de 25 de junho de 1986: Dispõe sobre a regulamentação do exercício da Enfermagem e dá outras providências. Brasília. http://www.cofen.gov.br/lei-n-749886-de-25-de-junho-de-1986_4161.html.

Conceição, G. M. N. (2015). Conhecimento dos enfermeiros de unidades de terapia intensiva adulto sobre as reações transfusionais. Trabalho de Conclusão de Curso (TCC) - Curso de Graduação em Enfermagem, Universidade Federal do Rio Grande do Sul, Porto Alegre. https://lume.ufrgs.br/bitstream/handle/10183/135489/000987141.pdf?sequence=1\&isAllowed=y.

Costa, M. L. A. S., Merighi, M. A. B., \& Jesus, M. C. P. (2008). Ser enfermeiro tendo sido estudante-trabalhador de enfermagem: um enfoque da fenomenologia social. Acta paulista de enfermagem, 21(1), 17-23. https://www.scielo.br/j/ape/a/DR7LP88VhQs4r8zXXLmLwYp/?format=pdf\&lang=pt.

Estrela, C. (2018). Metodologia Científica: Ciência, Ensino, Pesquisa. Editora Artes Médicas.

Ferreira, E. B., Santos, V. G. D. S., da Silva, F. P., da Silva, R. A., de Souza, C. F. Q., Da Costa, V. C., ... \& Guimarães, T. M. R. (2021). Hemovigilância: conhecimento da equipe de enfermagem sobre reações transfusionais. Enfermagem em http://revista.cofen.gov.br/index.php/enfermagem/article/view/4479.

Ferreira, O., Martinez, E. Z., Mota, C. A., \& Silva, A. M. (2007). Avaliação do conhecimento sobre hemoterapia e segurança transfusional de profissionais de enfermagem. Revista Brasileira de hematologia $\quad$ e 29(2), https://www.scielo.br/j/rbhh/a/SZmkpxYmf3YDPQrXRvrDVcq/?format=pdf\&lang=pt.

Fidlarczyk, D., \& Ferreira, S. S. (2008). Enfermagem em Hemoterapia. MedBook. Editora Científica Ltda.

Fontana, R. T., \& Brigo, L. (2012). Estudar e trabalhar: percepções de técnicos de enfermagem sobre esta escolha. Escola Anna Nery, 16(1), 128-133. https://www.scielo.br/j/ean/a/g6cVQ9Rrv5YVNMT8g7CDxVD/?format=pdf\&lang=pt.

Lopes, M. J. M., \& Leal, S. M. C. (2005). A feminização persistente na qualificação profissional da enfermagem brasileira. Cadernos Pagu, (24), 105-125. https://www.scielo.br/j/cpa/a/W4mKrfz7znsdGBdJxMHsGPG/?format=pdf\&lang=pt.

Mattia, D., \& Andrade, S. R. (2016). Cuidados de enfermagem na transfusão de sangue: um instrumento para monitorização do paciente. Texto \& Contexto Enfermagem, 25(2). https://www.scielo.br/j/tce/a/pDt9MgrD4SczNMRGNmzVyBt/?lang=en.

Silva, K. F. N., Soares, S., \& Iwamoto, H. H. (2009). A prática transfusional e a formação dos profissionais de saúde. Revista Brasileira de Hematologia e Hemoterapia, 31(6), 421-426. https://www.scielo.br/j/rbhh/a/xRDks4LwvsDqXSNXyj5sr6Q/?format=pdf\&lang=pt. 
Research, Society and Development, v. 11, n. 2, e55111225945, 2022

(CC BY 4.0) | ISSN 2525-3409 | DOI: http://dx.doi.org/10.33448/rsd-v11i2.25945

Silva, M. A., Torres, G. V., Melo, G. S. M., Costa, I. K. F., Tiburcio, M. P., \& Farias, T. Y. A. (2009). Conhecimento acerca do processo transfusional da equipe de enfermagem da uti de um hospital universitário. Ciência, Cuidado e Saúde, 8(4), 571-578. https://periodicos.uem.br/ojs/index.php/CiencCuidSaude/article/view/9676/5389.

Souza, G. F., Nascimento, E. R. P., Lazzari, D. D., Böes, A. A., Iung, W., \& Bertoncello, K. C. (2014). Boas práticas de enfermagem na unidade de terapia intensiva: cuidados durante e após a transfusão sanguínea. Revista Mineira de Enfermagem, 18(4), 939-954. http://www.reme.org.br/artigo/detalhes/974.

Tavares, J. L., Barichello, E., Mattia, A. L. de, \& Barbosa, M. H. (2015). Fatores associados ao conhecimento da equipe de enfermagem de um hospital de ensino sobre hemotransfusão. $\quad$ Revista Latino-Americana de Enfermagem, $\quad 23(4), \quad 595-602$. https://www.scielo.br/j/rlae/a/PYh44nMnnyQqnSkv6B3sTnt/?format=pdf\&lang=pt.

Vieira, M. S. (2012). Conhecimento da equipe enfermagem sobre hemoterapia. Trabalho de Conclusão de Curso (TCC) - Curso de Graduação em Enfermagem, Centro Universitário Univates, Lageado. https://www.univates.br/bdu/bitstream/10737/417/1/MarinaVieira.pdf. 